\title{
Effects of Entanglement on Off-diagonal Geometric Phases
}

\author{
H. T. Cui, L. C. Wang, X. X. Yi \\ Department of Physics, Dalian University of Technology, Dalian 116024, China
}

December 23, 2018

\begin{abstract}
The effect of entanglement on off-diagonal geometric phases is investigated in the paper. Two spin- $1 / 2$ particles in magnetic fields along the $y$ direction are taken as an example. Three parameters (the purity of state $r$, the mixing angle $\theta$ and the relative phase $\beta$ ) are chosen to characterize the initial states. The nodal points at which the usual geometric phases disappear are calculated and illustrated as a function of the three parameters.
\end{abstract}

PACS numbers: 03.65.Vf, 03.65.Ud

When a quantum system is transported around a curve $C$ in parameter space, in addition to the dynamical phase, a geometric phase can be developed which is path independent and determined only by the geometric nature of the Hilbert space. This geometric phase was first studied by Berry for a quantal system transporting adiabatically on a closed loop [1]. The generalization to the case of mixed states was given by Sjöqvist et al. [2] based on Mach-Zender interferometry, and was experimentally tested in different systems [3]. Besides, the study on geometric phase has also been extended to non-Abelian case for pure states [4] and mixed states [5] as well as open systems 6 . Recently Bhandari has pointed out that the definition for geometric phase in mixed state fails when the interference fringes disappear [7. This can be explained as the disappearance (appearance) of the geometric phase (off-diagonal geometric phase). The definition of off-diagonal geometric phase(OP) was first given by Manini et al. for pure states in adiabatic evolutions [8], and then was generalized to non-adiabatic situation [9] and in mixed states [10]. Most recently the off-diagonal geometric phase was studied in degenerate case [11] and in bipartite systems 12 .

On the other hand, entanglement as a property in quantum systems is proved to be useful in quantum information processing. Furthermore, it was found that geometric phase may be used to design quantum logic gates. These facts together give rise to the question of what the effect of entanglement on the (off-diagonal) geometric phase 13. We will try to answer this question in the paper.

The off-diagonal geometric phase is complementary to the usual geometric phase, i.e., when the geometric phase is undefined because the initial state evolves to its orthogonal states, the off-diagonal geometric phase would provide the phase information of state in time evolution 8 . In the case of adiabatic evolution, this can be understood as the appearing of a diabolic point in the parameter space, at which two orthogonal states have the same eigenenergy. Thus, the system initially in one of the orthogonal states may evolve to another one via this point. In this case the diagonal phase is undefined and the off-diagonal phase solely characterizes the phase change in the evolution and can be written as $[8]$,

$$
\gamma_{i j}=\arg \left(\sigma_{i j} \sigma_{j i}\right)
$$


where $\sigma_{i j}=\Phi\left(\left\langle\phi_{i}\left|U^{\|}(t)\right| \phi_{j}\right\rangle\right)$ with $U^{\|}(t)$ denoting a parallel evolution. $\Phi(z)=z /|z|$ for complex $z \neq 0$.

In what follows, we will discuss the effect of entanglement on the off-diagonal geometric phase. To this end, we choose initial state as

$$
\rho(0)=\sum_{k=1}^{N} \lambda_{k}|k\rangle\langle k|,
$$

where $|k\rangle(k=1, \ldots, M, M$ denotes the dimension of the Hilbert space) are eigenvectors of $\rho(0)$ with corresponding eigenvalues $\lambda_{k}$. Because of entanglement, the initial state Eq. (2) cannot be expressed as the direct product of the subsystem density operators. For two-qubit systems one can use Peres' condition [16] to judge whether $\rho$ is entangled or not. Under parallel evolution $U^{\|}(t)$, one can define GP as

$$
\gamma_{G P}=\arg \operatorname{Tr}\left[U^{\|}(t) \rho(0)\right]
$$

In general case, the nodal points, defined as those points in the parameter space where $\gamma_{G P}$ is undefined, are obtained as the solutions of

$$
\operatorname{Tr}\left[U^{\|}(t) \rho(0)\right]=0 .
$$

If the equation above is satisfied, we say that $\rho(0)$ and $\rho(t)=U(t) \rho(0) U^{\dagger}(t)$ are "orthogonal" to each other, where $U(t)$ stands for the time evolution operator for the system. More "orthogonal" states can be found by solving Eq. (44). This definition of "orthogonality" between two different density matrices is universal since it includes not only the case of the permutation of eigenstates in [10], but also the case of coherent superposition of eigenstates, that is, for example, the initial state is in a eigenstate $|k\rangle$, then the "orthogonal" state may be $\left|k^{\prime}\right\rangle=\sum_{l} \lambda_{l}|l\rangle$, in which $l \neq k$. Consequently, one can define OP as

$$
\gamma_{O P}^{(n)}=\arg \operatorname{Tr}\left[U^{\|}\left(t_{n-1}\right) \sqrt{\rho(0)} \prod_{l=1}^{n-1} U^{\|}\left(t_{n-1}\right) \sqrt{\rho\left(t_{l}\right)}\right],
$$

in which $\rho\left(t_{l}\right)$ is "orthogonal" to $\rho(0)$ and can be obtained by solving Eq. (4). So far we have presented general definitions for nodal points and off-diagonal geometric phases; exact expressions for nodal points and off-diagonal geometric phases depend on the detail of the initial states and the dynamics.

In the remaining of this paper, we will present a specific example to discuss OP and the effect of entanglement. For this purpose, we first consider a system consisting of two non-interacting spin- $1 / 2$ particles in an external magnetic field along the $y$ direction. The Hamiltonian reads $(\hbar=1)$

$$
H=\frac{\omega_{1}}{2} \sigma_{y}^{1}+\frac{\omega_{2}}{2} \sigma_{y}^{2}
$$

where $\sigma_{y}^{i}(i=1,2)$ are Pauli operators for the particle $i, \omega_{i}(i=1,2)$ represent the procession frequency. We notice that the off-diagonal item in the Hamiltonian Eq. (6) can lead to the transition between different eigenstates of $\sigma_{z}^{i}(\mathrm{i}=1,2)$. So under the time evolution governed by $H$ in Eq. (6), a state may evolve to its orthogonal states and hence the corresponding GP is undefined. Suppose that the initial state takes the form,

$$
\rho(0)=\frac{1-r}{4} I_{4}+r|\Phi\rangle\langle\Phi|,
$$


where $r \in(0,1]$ determines the purity of the mixed state. And we take $|\Phi\rangle$ from the following states,

$$
\begin{aligned}
|\varphi\rangle & =\cos \theta e^{i \beta}|11\rangle_{1,2}+\sin \theta e^{-i \beta}|00\rangle_{1,2} \\
|\psi\rangle & =\cos \theta e^{i \beta}|10\rangle_{1,2}+\sin \theta e^{-i \beta}|01\rangle_{1,2}
\end{aligned}
$$

where the mixing angle $\theta$, and the relative phase $\beta$ together determine the degree of entanglement. $|1(0)\rangle_{i}(i=1,2)$ is the eigenstate of the Pauli operator $\sigma_{z}^{i}(\mathrm{i}=1,2)$. When $\theta= \pm \pi / 4$ and $\beta=0$, the states Eq. (8) are the well known Bell states and correspondingly Eq.(17) gives the Werner state [17. The Werner state plays an important role in quantum information processing, in particular in quantum communication via noisy channels [18] and in the quantum distillation scheme 19. This initial state Eq. (17) includes all possible cases, such as pure or mixed states and maximal or non-maximal entangled states. One should note that these initial mixed states are triplet-degenerate.

Now we are in a position to discuss OP. Recently an experimental scheme for verifying second order $\operatorname{OP}(n=2$ in Eq. (51) ) has been proposed[10] based on the Franson-type interferometer, in which an entangled photon pair is produced by using spontaneous-down-conversion 20. This scheme can be used to prepare the initial state Eq. (7). First let us study the nodal structure for GP given by Eq. (4) under parallel transportation. The parallel transportation can be realized by imposing $[5]$

$$
U^{\|}(t)=U(t) V(t)
$$

where $U(t)$ is the unitary time-evolution operator and the elements of the blocked matrix $V(t)$ are defined in this model as

$$
\begin{aligned}
& V_{\mu \nu}=\left\langle\mu\left|e^{i t \sum_{\mu^{\prime}, \nu^{\prime}}\left\langle\mu^{\prime}|H| \nu^{\prime}\right\rangle\left|\mu^{\prime}\right\rangle\left\langle\nu^{\prime}\right|}\right| \nu\right\rangle,|\mu\rangle,|\nu\rangle,\left|\mu^{\prime}\right\rangle,\left|\nu^{\prime}\right\rangle \in\{\text { degenerate subspace }\} \\
& V_{k k}=e^{i\langle k|H| k\rangle t},|k\rangle \in\{\text { the remaining space }\}
\end{aligned}
$$

where $|\mu\rangle,|\nu\rangle,\left|\mu^{\prime}\right\rangle,\left|\nu^{\prime}\right\rangle,|k\rangle$ are the eigenstates of $\rho(0)$, and the interference terms between the degenerate space and the other space are set to be zero in order to keep the parallel transport in the degenerate space. We would like to note that the parallel transport operator $U^{\|}(t)$ depends on the initial state. So, different initial state leads to different nodal structure. The dependence of the nodal structure on the parameters of $r, \theta$ and $\beta$ can be obtained by solving Eq. (4). In order to simplify the tedious expression, we set $\omega_{1}=\omega_{2}=\omega$ and $\omega t=\pi$. The results then are reduced to

$$
\begin{gathered}
\operatorname{Tr}\left[U_{1}^{\|}(t) \rho_{1}\right]=\frac{1+3 r}{4} \cos 2 \beta \sin 2 \theta+\frac{1-r}{4}\left[1-(1+\cos 2 \beta \sin 2 \theta) \cos \frac{\pi}{2} \sqrt{2+2 \cos 2 \beta \sin 2 \theta}\right], \\
\operatorname{Tr}\left[U_{2}^{\|}(t) \rho_{2}\right]=-\frac{1+3 r}{4} \cos 2 \beta \sin 2 \theta+\frac{1-r}{4}\left[1-(1-\cos 2 \beta \sin 2 \theta) \cos \frac{\pi}{2} \sqrt{2-2 \cos 2 \beta \sin 2 \theta}\right],
\end{gathered}
$$

where

$$
\begin{aligned}
& \rho_{1}=\frac{1-r}{4} I_{4}+r|\varphi\rangle\langle\varphi|, \\
& \rho_{2}=\frac{1-r}{4} I_{4}+r|\psi\rangle\langle\psi|,
\end{aligned}
$$

The results show that there exist some common nodal points for the two initial states with $r=1$ and $\cos 2 \beta=0$ or $r=1$ and $\sin 2 \theta=0$, which indicates that the common nodal points lie in separable pure states. Note that the nodal points given by Eqs. (11) are a function of $\theta, \beta$ with period of $\pi$, then we may plot the nodal points only within $[0, \pi]$ for both $\theta$ and $\beta$. The 
(a)

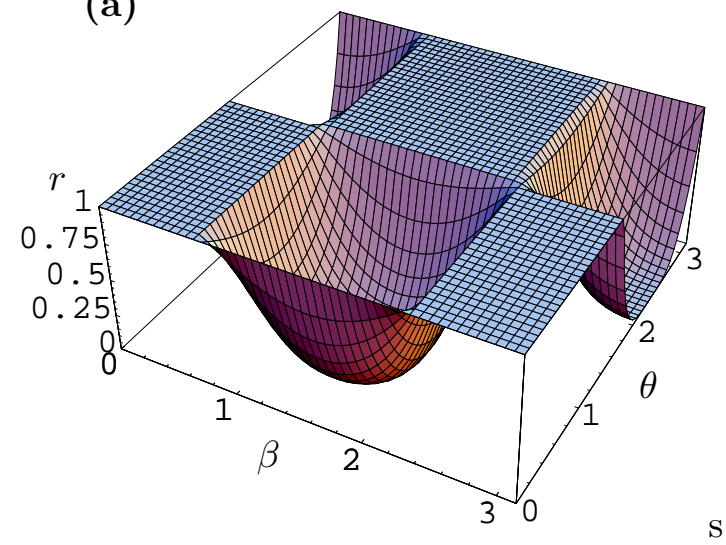

(b)

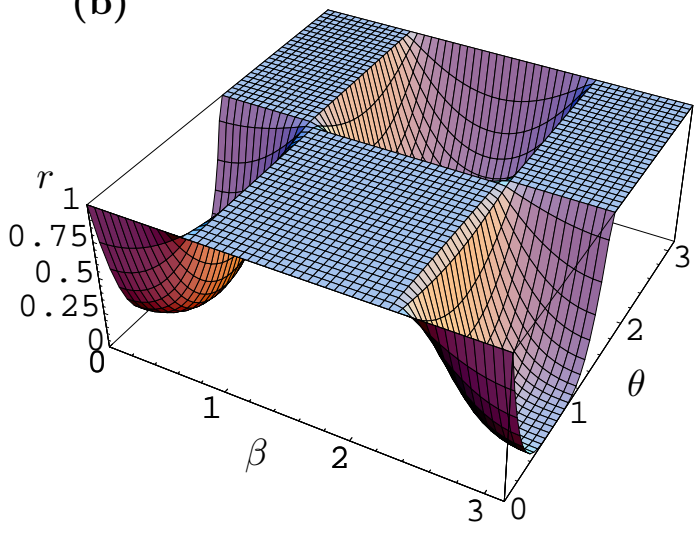

Figure 1: The nodal structure of GP for mixed states $\rho_{1}$ (a) and $\rho_{2}$ (b) vs $\beta[$ Arc], $\theta[$ Arc] with $\omega_{1} t=\omega_{2} t=\pi$.

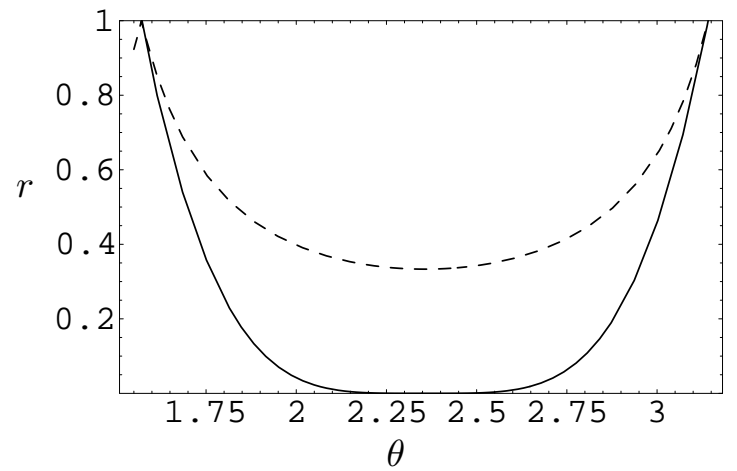

Figure 2: Comparison between the nodal structure and entanglement in the initial state. The dashed line denotes $r=\frac{1}{1+2|\sin 2 \theta|}$. We only draw for $\rho_{1}$ with $\beta=0$ and the other parameters are the same as those in Fig 1. Similar conclusion can also be found for $\rho_{2}$.

detailed dependence of the nodal structure on the three parameters was shown in Fig 1 It is clear that there are more nodal points in mixed states than that in pure states. Furthermore we find that the nodal points appear only with $0<r \leq \frac{1}{1+2|\sin 2 \theta|}$ (see Fig[2), that is exactly the condition for $\rho_{1,2}$ being separable states. However, we find that when the initial state is a Werner state, no nodal points exist in the parameter space for any $r \in(0,1]$.

Next let us study the two-indexes OP $(n=2)$. According to the definition of Eq. (5), we may write the two-indexes off-diagonal geometric phase as

$$
\gamma_{O P}^{(2)}=\arg \operatorname{Tr}\left[U^{\|}(t) \sqrt{\rho(0)} U^{\|^{\prime}}(t) \sqrt{\rho(t)}\right]
$$

where $\rho(t)=U(t) \rho(0) U^{\dagger}(t)$, and parallel evolution $U^{\|^{\prime}}(t)$ is different from the previous one. The same conditions, namely $\omega_{1}=\omega_{2}=\omega$, were chosen to simplify the expression. With this assumption, the two-indexes $\mathrm{OP}(n=2)$ is given by

$$
\gamma_{O P}^{(2)}\left(\rho_{1,2}\right)=\arg \left[\frac{1+r}{2}+\frac{1-r}{2} \cos \pi \sqrt{2 \pm \cos 2 \beta \sin 2 \theta}\right]
$$

Obviously the value of $\gamma_{O P}^{(2)}\left(\rho_{1,2}\right)$ is always zero when the diagonal geometric phase is undefined, independent of the initial state. This property is very different from the diagonal geometric phase, which depend not only on the initial state, but also on the dynamics of the system. It 
(a)

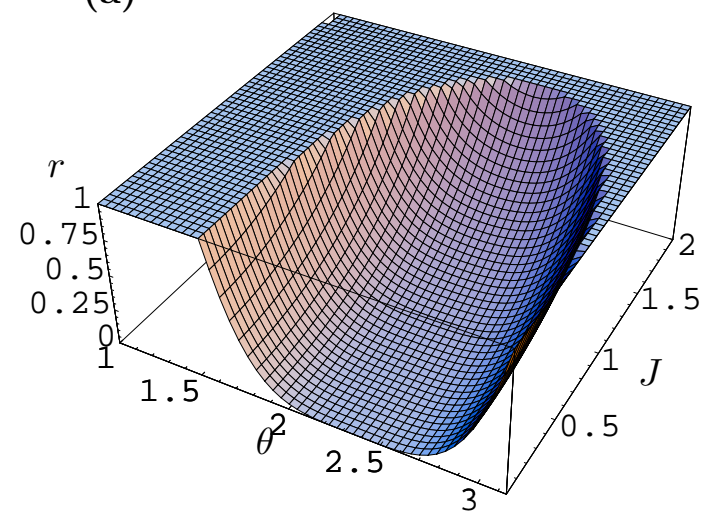

(b)

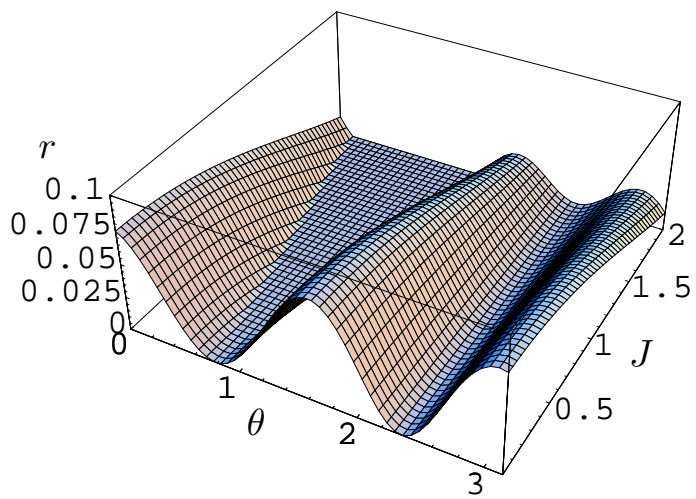

Figure 3: The nodal structure of GP for mixed states $\rho_{1}$ with inter-subsystem couplings vs $\theta[$ Arc] and the rescaled coupling constant $J=g / \omega$. (a) corresponds to the real part of Eq. (4) and (b) corresponds to the imaginary part of Eq. (4). $\omega_{1} t=\omega_{2} t=\pi$ and $\beta=0$ were chosen for this plot.

furthermore reflects the topology of the off-diagonal geometric phase, as the value of OP depends on the degeneracy [8]. In fact since the nodal structure for diagonal geometric phase appears only for unentangled state and there is no intra-coupling in our model, then OP is the sum over that of the two particles.

So far we only discuss the nodal structure of geometric phase without inter-subsystem couplings. Another question is how the nodal structure is affected by the inter-subsystem couplings. For this purpose we consider the Ising-type interaction,

$$
H_{I}=\frac{g}{4} \sigma_{z}^{1} \sigma_{z}^{2}
$$

In general the interaction tends to destroy the degeneracy or the level (avoided) crossing and we guess that the nodal structure should be compressed because of the couplings. After calculations we found that this is the casae, i.e., the intra-coupling tends to destroy the nodal structure and the nodal points only appear for special initial states with certain coupling constant. We do not try to list the results of our calculation because of the complicated expressions, the selected numerical results were shown in Fig. 3 From the figure we see that because of the inter-subsystem couplings, Eq. (4) is a complex number and hence in order to determine the nodal points, we have to let the real and imaginary part of the complex number to be zero simultaneously. Different from the free case, the nodal points appear only for the special values of $\theta$, displayed in Fig. 4 and only exist for weak couplings $(J \leq 0.28)$. Moreover we find that the separable condition for the initial state is required too. Besides when the initial state is a Werner state, there is no nodal point appearing. The two-index OP has been calculated and our calculations show that the values of OP are not always zero or $\pi$, this result comes from the intra-coupling and also depends on the degeneracy. Similar conclusions can be found for $\rho_{2}$.

In conclusion, we have discussed the entanglement effect on the off-diagonal geometric phase in mixed states by a general example and some novel results are presented. First, because of entanglement and mixture of the initial states, the nodal structure for GP have been changed greatly, as displayed in Fig. 11 We found that the nodal points only appear in region $0<r \leq$ $\frac{1}{1+2|\sin 2 \theta|}$ as displayed in Fig 2] which means the initial states are separable. Furthermore when the initial state is a Werner state, we found there is no nodal point for any $r \in(0,1]$. The two-indexes OP for the nodal points are shown to be zero, independent of the initial states. We 


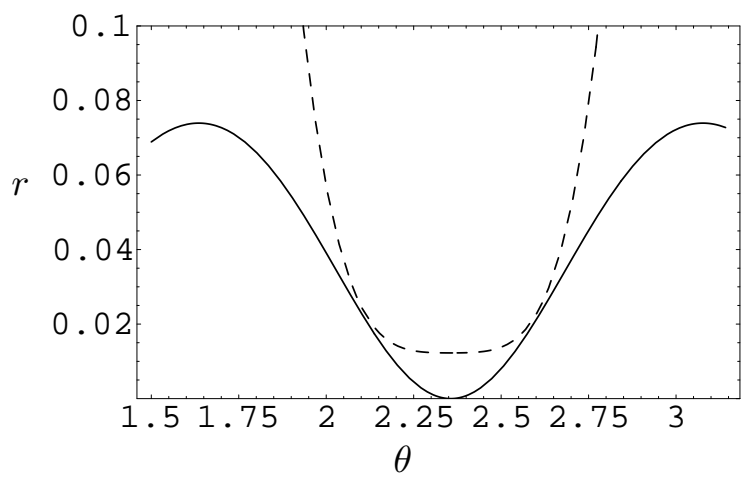

Figure 4: Detailed demonstration for the Fig 3. We have chosen $J=0.28$ and the other parameters is the same as that in Fig 3. The dashed line corresponds to (a) and solid line corresponds to (b) in Fig 3 With the decrease of $J$, the dashed line moves downward.

also extend this discussion to the case with inter-subsystem couplings. The results show that because of the coupling the nodal points for GP have been compressed greatly and only appear for some special initial state and small coupling constants. Besides we find that similar to the free case, there is no nodal point for the Werner state, implying that the Werner state is robust against the perturbation.

This work was supported by NSF of China under grants 10305002 and 60578014 .

\section{References}

[1] M. V. Berry, Proc. R. Soc. London A 392, 45(1984).

[2] E. Sjöqvist, A. K. Pati, A. Ekert, J. S. Anandan, M. Ericsson, D. K. L. Oi, V. Vedral, Phys. Rev. Lett. 85, 2845(2000).

[3] J. Du, et al., Phys. Rev. Lett. 91, 100403 (2003); M. Ericsson, et al., Phys. Rev. Lett. 94, 050401 (2005); J. Klepp, et al., Phys. Lett. A 342, 48 (2005); A. Ghosh and A. Kumar, Phys. Lett. A 349, 27 (2005).

[4] F. Wilczek, A. Zee, Phys. Rev. Lett. 52, 2111 (1984).

[5] K. Singh, et al., Phys. Rev. A. 67, 032016 (2003); D. M. Tong, et al., Phys. Rev. Lett. 93, 080405 (2004).

[6] M. Ericsson, et al., Phys. Rev. A 67, 020101 (2003); J. G. Peixoto de Faria, et al., Europhys. Lett. 62, 782 (2003); R. S. Whitney, Y. Gefen, Phys. Rev. Lett. 90, 190402 (2003); A. Carollo, et al., ibid, 90, 160402 (2003); K. P. Marzlin, et al., ibid, 93, 260402 (2004); I. Kamleitner, et al., Phys. Rev. A. 70, 044103(2004); R. S. Whitney, et al., Phys. Rev. Lett. 94, 070407 (2005); X. X. Yi, L. C. Wang, and W. Wang, Phys. Rev. A 71, 044101 (2005); X X Yi, D P Liu and W Wang, New J. Phys. 7, 222 (2005).

[7] R. Bhandari, Phys. Rev. Lett. 89, 268901(2002); J. Anandan, et al., Phys. Rev. Lett. 89, $268902(2002)$.

[8] N. Manini, F. Pistolesi, Phys. Rev. Lett. 85, 3067(2000).

[9] N. Munkunda, Arvind, E. Ercolessi, G. Marmo, G. Morandi, R. Simon, Phys. Rev. A., 67, 042114(2003). 
[10] S. Filipp, E. Sjöqvist, Phys. Rev. Lett. 90, 050403(2003); Phys. Rev. A. 68, 042112(2003).

[11] D. M. Tong, E. Sjöqvist, S. Filipp, L. C. Kwek, C. H. Oh, Phys. Rev. A, 71, 032106(2005).

[12] X. X. Yi, J. L. Chang, Phys. Rev. A., 70, 012108 (2004).

[13] The effect of entanglement on the geometric phase was studied already [14, 15, however the effect on the off-diagnoal geometric phase in particular for mixed states remains untouched.

[14] E. Sjöqvist, Phys. Rev. A. 62, 022109(2000).

[15] D. M. Tong, et al., Phys. Rev. A. 68, 022106(2003); J. Phys. A: Math. Gen. 36, 1149(2003).

[16] A. Peres, Phys. Rev. Lett. 77, 1413 (1996).

[17] R. F. Werner, Phys. Rev. A. 40, 4277 (1989).

[18] C. H. Bennett, D. P. DiVincenzo, J. A. Smolin, and W. K. Wootters, Phys. Rev. A 54, 3824(1996); C. H. Bennett, P. W. Shor, J. A. Smolin, and A. V. Thapliyal, Phys. Rev. Lett. 83, 3081(1999).

[19] C. H. Bennett, G. Brassard, S. Popescu, B. Schumacher, J. A. Smolin, and W. K. Wootters, Phys. Rev. Lett. 76 , 722(1996).

[20] P. G. Kwiat et al., Phys. Rev. A 60, R773(1999); A. G. White, D. F. V. James, P. H. Eberhard, and P. G. Kwiat, Phys. Rev. Lett. 83, 3103(1999). 